\title{
Polyhomomorphisms of locally compact groups
}

\author{
YuRY A. Neretin 1
}

Let $G$ and $H$ be locally compact groups with fixed two-side-invariant Haar measures. A polyhomomorphism $G \longmapsto H$ is a closed subgroup $R \subset G \times H$ with a fixed Haar measure, whose marginals on $G$ and $H$ are dominated by the Haar measures on $G$ and $H$. A polyhomomorphism can be regarded as a multi-valued map sending points to sets equipped with 'uniform' measures. For polyhomomorphsisms $G \longmapsto H, H \longmapsto K$ there is a well-defined product $G \longmapsto K$. The set of polyhomomorphisms $G \longmapsto H$ is a metrizable compact space with respect to the Chabauty-Bourbaki topology and the product is separately continuous. A polyhomomorphism $G \longmapsto H$ determines a canonical operator $L^{2}(H) \rightarrow L^{2}(G)$, which is a partial isometry up to scalar factor. As an example, we consider locally compact infinite-dimensional linear spaces over finite fields and examine closures of groups of linear operators in semigroups of polyendomorphisms.

\section{Polyhomomorphisms}

We consider only second-countable locally compact groups $G$, i. e., locally group having countable base of open subsets. Such groups admit left invariant metrics (see, e.g. [7, Theorem 8.3), so they are separable as metric spaces. They are complete topological groups in the sense of Weil-Bourbaki ([3], Corollary III.3.1). Such a group admits a unique up to a scalar factor left-invariant measure $\gamma(g)$ (the Haar measure), see [4, Theorem VII.1.1, 7, Theorem 8.3, 40, Theorem 26.4, 38, 24, Chapter A.

Since $G$ has a structure of complete metric space, the Borel structure on $G$ is standard (see, e. g., [10, Sect 12), as a Borel space $G$ is isomorphic to the line $\mathbb{R}$, or a countable set, or a finite set. As a space with measure the group $G$ is a Lebesgue space (see, e. g., [2], Chapter 10), as a space with measure $G$ can be isomorphic to an interval, the line $\mathbb{R}$, a countable or finite set .

Since we have a measure, we also have standard spaces of measurable functions on $G$ as $L^{2}(G)$. By $C_{c}(G)$ we denote the space of continuous functions on $G$ with compact support.

A locally compact group is unimodular if the Haar measure is two-side invariant (see [4, Subsect. VII.1.3-4).

Let $K \supset L$ be groups. Denote by $[K: L]$ the index, i.e., the number of elements in $K / L$.

For a set $X$ and subset $A \subset X$ we denote by $I_{A}(x)$ the indicator function of $A$, i.e., $I_{A}(x)=1$ if $x \in A$ and 0 otherwise.

1.1. Multiplicative relations. Let $X, Y$ be sets. A relation $X \rightrightarrows Y$ is a subset $R \subset X \times Y$. For two relations $R: X \rightrightarrows Y, S: Y \rightrightarrows Z$ we define their

\footnotetext{
${ }^{1}$ Supported by the grant FWF, P31591.
} 
product $S R: X \rightrightarrows Z$ as the set of all $(x, y) \in X \times Z$, for which there exists $y \in Y$ such that $(x, y) \in R,(y, z) \in S$. Clearly, this product is associative.

For a relation $R: X \rightrightarrows Y$ we define:

- the image im $R$ is the projection of $R$ to $Y$;

- the domain $\operatorname{dom} R$ is the projection of $R$ to $X$.

Define the pseudoinverse relation $R^{\square}: Y \rightrightarrows X$ as the same subset $R \subset X \times Y$ considered as a relation from $Y$ to $X$. Obviously,

$$
(T R)^{\square}=R^{\square} T^{\square} .
$$

For a subset $A \subset X$ we define its image $R A$ as the set of all $b \in Y$ such that there exists $a \in A$ satisfying $(a, b) \in R$.

REMARK. If $f: X \rightarrow Y$ is a map, then its graph $\Gamma(f) \subset X \times Y$ is a relation, $\operatorname{dom} \Gamma(f)=X$ and the projection map $\Gamma(f) \rightarrow X$ is injective.

A partial bijection $X \rightarrow Y$ is a bijective map of a subset $A \subset X$ to a subset $B \subset Y$. A relation $R: X \rightrightarrows Y$ is a partial bijection if the projection maps from $R$ to $X$ and $Y$ are injective.

Let $G, H$ be groups. A multiplicative relation $R: G \rightrightarrows H$ is a subgroup in $G \times H$. Clearly, a product of multiplicative relations is a multiplicative relation. For a multiplicative relation $R: G \rightrightarrows H$ we define

- the kernel as the intersection of $R$ with $G \subset G \times H$;

- the indefinity as the intersection of $R$ with $H \subset G \times H$.

The following statement is obvious.

Lemma 1.1 a) The kernel $\operatorname{ker} R$ is a normal subgroup in $\operatorname{dom} R$ and $\operatorname{indef} R$ is a normal subgroup in $\mathrm{im} R$.

b) A multiplicative relation $R$ determines a canonical isomorphism

$$
\iota(R): \operatorname{dom} R / \operatorname{ker} R \rightarrow \operatorname{im} R / \operatorname{indef} R .
$$

c) The subgroups ker $R$, indef $R$, $\operatorname{ker} R \times$ indef $R$ are normal in $R$.

We define a partial isomorphism $G \rightarrow H$ as a partial bijection between two subgroups $A \subset G, B \subset H$ sending products to products.

If groups $G, H$ are additive, then it is reasonable to say 'additive relation'. If they are linear spaces and $R$ is a subspace, we say 'linear relation'. Linear relations and additive relation are usual mathematical objects (see, e.g., 14, 22]), multiplicative relations are known but appear not too often, see, e.g., 34, Sect. 1.2 .

1.2. The category of polyhomomorphisms. Let $X$ be a space with measure $\xi$, let $Y$ be a set and $f$ be a map $X \rightarrow Y$. Recall that the image $v$ of the measure $\xi$ under the map $f$ is the measure on $Y$ defined by the condition $v(B):=\xi\left(f^{-1}(B)\right)$. 
Denote by $G^{\circ}\left(\right.$ resp. $\left.H^{\circ}\right)$ a unimodular group $G($ resp. $H$ ) with a fixed Haar measure $\gamma(g)$ (resp. $\eta(h)$ ). Denote by $\overleftarrow{\pi}$ the natural projection map $G \times H \rightarrow$ $G$, by $\vec{\pi}$ the projection map $G \times H \rightarrow H$ We say that a polyhomomorphism $R^{\circ}: G^{\circ} \longmapsto H^{\circ}$ is an object of one of the following types:

1. a closed subgroup $R \subset G \times H$ with a fixed Haar measure $\rho(r)$ such that the image of $\rho$ under $\overleftarrow{\pi}$ (respectively, $\vec{\pi}$ ) is dominated by $\gamma(g)$ (resp. by $\eta(h)$ );

2. the zero measure $0=0_{G, H}$ on $G \times H$.

Denote by $\operatorname{Polh}\left(G^{\circ}, H^{\circ}\right)$ the set of all polyhomomorphisms $G^{\circ} \longmapsto H^{\circ}$. Elements of this set automatically satisfy the following properties (so they can be included to the definition of polyhomomorphisms).

Proposition 1.2 Let $R^{\circ} \in \operatorname{Polh}\left(G^{\circ}, H^{\circ}\right)$ and $R$ the underlying multiplicative relation. Then

a) The subgroups $\operatorname{ker} R \subset G$, indef $R \subset H$ are compact.

b) The subgroups $\operatorname{dom} R \subset G$, im $R \subset H$ are open

c) The group $R$ is unimodular.

d) The image of the measure $\rho(r)$ under the projection $\overleftarrow{\pi}: R \rightarrow G$ is the measure on $\operatorname{dom} G$ having the form $\alpha \gamma(g)$, where

$$
\alpha=\alpha\left(R^{\circ}\right)
$$

is a constant such that $0<\alpha \leqslant 1$. Similarly, the image of $\rho(r)$ under $\vec{\pi}$ is the measure on $\operatorname{im} R$ of the form $\beta \eta(h)$, where

$$
\beta=\beta\left(R^{\circ}\right)
$$

is a constant satisfying the condition $0<\beta \leqslant 1$.

The proof is contained in Subsect. 2.1,

Next, let $R^{\circ} \in\left(G^{\circ}, H^{\circ}\right), T^{\circ} \in \operatorname{Polh}\left(H^{\circ}, K^{\circ}\right)$ be two nonzero polyhomomorphisms. We define their product $S^{\circ}=T^{\circ} R^{\circ} \in \operatorname{Polh}\left(G^{\circ}, K^{\circ}\right)$ as follows:

1) a multiplicative relation $S$ is $S:=T R$;

2 ) we normalize the Haar measure on $S$ in the terms of its images under the projections to $G$ and $K$ :

$$
\begin{aligned}
\alpha\left(S^{\circ}\right) & =\frac{\alpha\left(R^{\circ}\right) \alpha\left(T^{\circ}\right)}{[\operatorname{indef} R:(\operatorname{indef} R \cap \operatorname{dom} T)]} ; \\
\beta\left(S^{\circ}\right) & =\frac{\beta\left(R^{\circ}\right) \beta\left(T^{\circ}\right)}{[\operatorname{ker} T:(\operatorname{ker} T \cap \operatorname{im} R)]} .
\end{aligned}
$$

A product of a zero polyhomomorphism and any polyhomomorphism is zero,

$$
0_{H, K} R^{\circ}=0_{G, K}, \quad T^{\circ} 0_{G, H}=0_{G, K}, \quad 0_{H, K} 0_{G, H}=0_{G, K} .
$$


Lemma 1.3 This product is well defined and associative, i. e., for any $G^{\circ}, H^{\circ}$, $K^{\circ}, L^{\circ}$ and any

$$
R^{\circ} \in \operatorname{Polh}\left(G^{\circ}, H^{\circ}\right), \quad T^{\circ} \in \operatorname{Polh}\left(H^{\circ}, K^{\circ}\right), \quad S^{\circ} \in \operatorname{Polh}\left(K^{\circ}, L^{\circ}\right)
$$

we have

$$
\left(S^{\circ} T^{\circ}\right) R^{\circ}=S^{\circ}\left(T^{\circ} R^{\circ}\right)
$$

REMARK. In fact the definition of a product becomes a theorem if we consider polyhomomorphisms as special cases of polymorphisms, see below Subsect. 1.51 .6 .

Thus we get a category of polyhomomorphisms. Objects $G^{\circ}$ of this category are unimodular locally compact groups equipped with fixed Haar measures. The set of morphisms from $G^{\circ}$ to $H^{\circ}$ is $\operatorname{Polh}\left(G^{\circ}, H^{\circ}\right)$.

For $R^{\circ} \in \operatorname{Polh}\left(G^{\circ}, H^{\circ}\right)$ denote by $\left(R^{\circ}\right)^{\square}$ the same subgroup in $G \times H$ with the same Haar measure considered as a subgroup in $H \times G$. Obviously, $\left(T^{\circ} R^{\circ}\right)^{\square}=\left(R^{\circ}\right)^{\square}\left(T^{\circ}\right)^{\square}$.

1.3. Comments to the definition. Here we briefly discuss, what means this definition for some natural classes of locally compact groups.

Finite groups. Let groups $G, H$ be finite. Normalize measures assuming that each element has measure 1. A subgroup $R \subset G \times H$ can be arbitrary, we must equip it with a uniform measure. Assuming that a measure of each element of the group $R$ is $\mathfrak{r}$, we get the following inequalities for $\mathfrak{r}$ :

$$
\alpha\left(R^{\circ}\right)=\mathfrak{r} \cdot \# \text { indef } R \leqslant 1, \quad \beta\left(R^{\circ}\right)=\mathfrak{r} \cdot \# \operatorname{ker} R \leqslant 1,
$$

the symbol $\# X$ denotes the number of elements of a set $X$.

The category of polyhomomorphisms is a kind of a 'central extension' of the category of multiplicative linear relations 2 . Let explain an origin of this extension with an example of finite groups. Denote by $\ell^{2}(G)$ the space of functions on $G$ equipped with the $\ell^{2}$-inner product. Notice that any homomorphism $\rho: G \rightarrow H$ determines an operator

$$
\Pi_{*}(\rho): \ell^{2}(H) \rightarrow \ell^{2}(G)
$$

by the formula

$$
\Pi_{*}(\rho) f(g):=f(\rho(g)) .
$$

If $\sigma$ is a homomorphism $H \rightarrow K$, then

$$
\Pi_{*}(\rho \circ \sigma)=\Pi_{*}(\rho) \Pi_{*}(\sigma) .
$$

For a multiplicative relation $R: G \rightrightarrows H$ we can define the following operator $\ell^{2}(H) \rightarrow \ell^{2}(G)$ :

$$
\Pi_{*}(R) f(g):=\sum_{h \in H:(g, h) \in R} f(h) .
$$

\footnotetext{
${ }^{2}$ See a formal definition in [21 Subsect. I.8.6]. Notice that categories of linear relations arising in representation theory appear together with their central extensions, see [21], [19].
} 
It easy to show that for multiplicative relations $R: G \rightrightarrows H, T: H \rightrightarrows K$ the following identity holds

$$
\begin{aligned}
& \Pi_{*}(R) \Pi_{*}(T)=\#(\operatorname{ker} T \cap \operatorname{indef} R) \cdot \Pi_{*}(T R)= \\
= & \frac{\# \operatorname{indef} T \cdot \#(\operatorname{indef} R \cap \operatorname{dom} T)}{\# \operatorname{indef} T R} \Pi_{*}(T R)=\frac{\# \operatorname{ker} R \cdot \#(\operatorname{ker} T \cap \operatorname{im} R)}{\# \operatorname{ker} T R} \Pi_{*}(T R) .
\end{aligned}
$$

As above, define a uniform measure on $R$ and modify operators assuming

$$
\Pi\left(R^{\circ}\right):=\mathfrak{r} \cdot \Pi_{*}(R) .
$$

It is easy to see that

$$
\Pi(R) \Pi(T)=\Pi\left(T^{\circ} R^{\circ}\right) .
$$

An extension of this construction to arbitrary polyhomomorphisms is discussed below in Subsect. 1.7.

Discrete Groups. If $G, H$ are discrete (countable), then we have an additional condition for $R$ : the groups ker $R$ and indef $R$ must be finite. Notice also, that this condition is necessary and sufficient for the boundedness of the operator (1.3).

LIE GROUPS. For connected Lie groups the notion of polyhomomorphism gives little new in comparation with homomorphisms. By definition, connected groups do not have proper open subgroups, on other hand there are few compact normal subgroups in Lie groups. Therefore it remains little possibilities to satisfy conditions of Proposition 1.2 a-b and Lemma 1.1]a.

For a reader familiar with theory of Lie groups, we present a typical example of a polyhomomorphism between semisimple groups. Consider the group $G:=\mathrm{SL}(2, \mathbb{R})$ (the group of real matrices of order 2 with determinant 1, it is homotopically equivalent to the circle, so its fundamental group is $\mathbb{Z}$ ). Denote by $G^{\sim n}$ its $n$-sheeted covering. The group $G^{\sim 6}$ embeds to $G^{\sim 2} \times G^{\sim 3}$ and can be regarded as a multiplicative relation, it remains to normalize a Haar measure on $G^{\sim 6}$ in some way.

The case of tori gives more possibilities. Recall that a torus is a quotient $\mathbb{R}^{n} / \mathbb{Z}^{n}$, we equip it with a probabilistic Haar measure. We need closed subgroups in $\mathbb{R}^{n} / \mathbb{Z}^{n} \times \mathbb{R}^{m} / \mathbb{Z}^{m}$. They can be easily described. Namely let $L$ be a linear subspace in $\mathbb{R}^{n} \times \mathbb{R}^{m}$ determined by equations with integer coefficients,

$$
\left\{\sum_{i=1}^{n} p_{\alpha}^{i} x_{i}+\sum_{j=1}^{m} q_{\alpha}^{j} y_{j}=0, \quad \text { where } \alpha=1, \ldots, k,\right.
$$

or, in a matrix form $P x+Q y=0$. The image of $L$ under the map $\mathbb{R}^{n} \times \mathbb{R}^{m} \rightarrow$ $\mathbb{R}^{n} / \mathbb{Z}^{n} \times \mathbb{R}^{m} / \mathbb{Z}^{m}$ is a closed subgroup in the product of tori, and all closed subgroups have such a form.

Next, consider a finitely generated subgroup $\Gamma$ in a linear space $\mathbb{Q}^{k}$ over rationals. Take the set $L_{\Gamma}$ of vectors in $\mathbb{R}^{n+m}$, satisfying the condition $P x+Q y \in$ 
$\Gamma$ and consider its projection to $\mathbb{R}^{n} / \mathbb{Z}^{n} \times \mathbb{R}^{m} / \mathbb{Z}^{m}$. This construction gives us all closed subgroups in a product of tori.

To get a polyhomomorphism, we need the surjectivity of the projections of $L$ to $\mathbb{R}^{n}$ and $\mathbb{R}^{m}$. Notice that in this case the denominators in formulas (1.1)-(1.2) equal 1. Therefore nothing prevents us without loss of substance set $\alpha=\beta=1$ for all polyhomomorphisms.

TOTALLY DisCONNECTED NON-DISCRETE GROUPS. There are lot of totally disconnected locally compact groups arising in various branches of mathematics (for instance, $p$-adic and adelic groups, Galois groups equipped with the Krull topology, groups of automorphisms and spheromorphisms of trees, some infinitedimensional groups over finite fields.

Notice that each group $G$ of this type has 'many' polyendomorphisms (i.e., polyhomomorphisms to itself). We present mass examples:

1) Let $\Omega \subset G$ be an open subgroup. We take the subgroup $R \subset G \times G$ consisting of points of the form, $(g, g)$, where $g$ ranges in $\Omega$. Evidently, $R \simeq \Omega$, and this determines a Haar measure on $\Omega$.

2) Take an open subgroup $\Omega \subset G$ and a normal compact open subgroun 3 $L \subset \Omega$. We take $R \subset G \times G$ consisting of elements of the form

$$
(l g, g), \quad \text { where } g \in \Omega, l \in L .
$$

It remains to equip $R$ with a Haar measure (and multiply it by a sufficiently small factor if this is necessary).

3) Any automorphism $\theta$ of a group $G$ (for instance, an interior automorphism) generates a polyhomomorphism $G^{\circ} \longmapsto G^{\circ}$. As $R$ we take a graph of the map $\theta$ and equip it with an appropriate Haar measure4.

Further, we can multiply polyhomomorphisms of types 1)-3).

1.4. The convergence of polyhomomorphisms. We define a convergence in $\operatorname{Polh}\left(G^{\circ}, H^{\circ}\right)$ as the weak convergence of measures (see, e. g., [2], Sect.8.1) on $G \times H$. Namely, let $R_{j}^{\circ}=\left(R_{j}, \rho_{j}\right), R^{\circ}=(R, \rho)$ be polyhomomorphisms $G \longmapsto H$. A sequence $R_{j}^{\circ}: G^{\circ} \longmapsto H^{\circ}$ converges to $R^{\circ}: G^{\circ} \longmapsto H^{\circ}$ if for any functions $\varphi \in C_{c}(G)$ and $\psi \in C_{c}(H)$ we have the converges of integrals

$$
\int_{R_{j}} \varphi(\overleftarrow{\pi}(r)) \psi(\vec{\pi}(r)) d \rho_{j}(r) \rightarrow \int_{R} \varphi(\overleftarrow{\pi}(r)) \psi(\vec{\pi}(r)) d \rho(r)
$$

A sequence $R_{j}^{\circ}$ converges to $0_{G, H}$ if for any $\varphi, \psi$ we have the convergence

$$
\int_{R_{j}} \varphi(\overleftarrow{\pi}(r)) \psi(\vec{\pi}(r)) d \rho_{j}(r) \rightarrow 0
$$

\footnotetext{
${ }^{3}$ A totally disconnected locally compact group has a fundamental system of neighborhoods of the unit consisting of open compact subgroup. If the group is compact, then these subgroups can be chosen normal, see [7. Theorems 7.5-7.7]. This implies that there is a countable set of open subgroups in $\Omega$; for a fixed open compact subgroup $\Omega$ we have a countable set of open normal subgroups.

${ }^{4}$ Generally speaking, the measures $\gamma(g)$ and $\gamma(\theta(g))$ differs by a scalar factor $c$, see. e.g., [4], Subsect. VII.1.4. If $c \neq 1$, then the coefficients $\alpha\left(R^{\circ}\right)$ and $\beta\left(R^{\circ}\right)$ are different.
} 
REMARK. We can define the convergence in the following equivalent way: $R_{j}^{\circ}$ converges to $R^{\circ}$ if for any $\theta \in C_{c}(G \times H)$ we have the convergence

$$
\int_{R_{j}} \theta(r) d \rho_{j}(r) \rightarrow \int_{R} \theta(r) d \rho(r) .
$$

Similarly, $R_{j}^{\circ}$ converges to $0_{G, H}$ if such sequences of integrals converge to 0 . $\otimes$

Proposition 1.4 a) This convergence is metrizable and sets $\operatorname{Polh}\left(G^{\circ}, H^{\circ}\right)$ are compact.

b) The product of polyhomomorphisms is separately continuous.

This convergence is a rephrasing the Chabauty-Bourbaki convergence of subgroups in locally compact groups, see Bourbaki [4, Sect VIII.5, Bourbaki normalizes Haar measures on each subgroup, we allow to vary scalar factors. The compactness is Theorem VIII.5.1 of Bourbaki.

Remark. Convergence $R_{j}^{\circ} \rightarrow R^{\circ}$ implies a convergence of subsets $R_{j} \rightarrow R$. There are many non-equivalent definitions of convergences on sets of closed subsets of topological or metric spaces, see, e. g., [15]. Our space $G \times H$ is locally compact and reasonable topologies coincide. For instance (see Bourbaki [3, Subsect. VIII.5.6) we can take a left invariant metric on $G \times H$ compatible with the topology and say that a sequence $R_{j}$ of closed subgroups converges to $R$ if for each $\varepsilon>0$ for any compact set $K \subset G \times H$ for sufficiently large $j$ the set $K \cap R$ is contained in the $\varepsilon$-neighborhood of $R_{j}$ and $K \cap R_{j}$ is contained in the $\varepsilon$-neighborhood of of $R$. See [1] on a way to define a metric on this space. $\nabla$

REMARK. A convergence $R_{j}^{\circ} \rightarrow R^{\circ}$ does not implies convergences ker $R_{j} \rightarrow$ $\operatorname{ker} R_{j}, \operatorname{dom} R_{j} \rightarrow \operatorname{dom} R, \alpha\left(R_{j}^{\circ}\right) \rightarrow \alpha\left(R_{j}^{\circ}\right)$, etc. However, we have some semicontinuities. If ker $R_{j}$ contain some subgroup $L \subset G$ starting some $j$, then $\operatorname{ker} R$ contains $L$. If dom $R_{j}$ are contained in a certain subgroup $M \subset G$ starting some $j$, then $\operatorname{dom} R$ is contained in the same subgroup. If $\alpha\left(R_{j}^{\circ}\right) \leqslant s$ starting some $j$, then $\alpha\left(R^{\circ}\right) \leqslant s$.

1.5. Polymorphisms. Preliminaries. See [18, 19, Sect VIII.4.

A. Category of polymorphisms. Recall that a space $X$ with a finite or $\sigma$-finite measure $\xi$ is a Lebesgue measure space if it is equivalent to a union of a finite or infinite interval of the line $\mathbb{R}$ and of a finite (may be, empty) or countable collection of points having positive measures.

REMARK. A locally compact group $G$ equipped with the Haar measure as a measure space is equivalent to

- a collection of points having equal positive measures if a group is discrete;

- a finite interval $(a, b) \subset \mathbb{R}$ if a group is compact and infinite;

- $\mathbb{R}$ otherwise.

Let $(X, \xi)$ and $(Y, v)$ be Lebesgue spaces. A polymorphism $\mu:(X, \xi) \longmapsto$ $(Y, v)$ is a measure $\alpha$ on $X \times Y$ such that projection of $\mu$ to $X$ is dominated by 
$\xi$ (i.e., for any subset $A \subset X$ of finite measure we have $\xi(A) \geqslant \mu(A \times Y)$ ) and the projection of $\mu$ to $Y$ is dominated by $v$. We admit zero measures. Denote the set of all polymorphisms $X \longmapsto Y$ by $\operatorname{Pol}(X, Y)$.

We regard a polymorphism as a 'multivalued maps' $X \rightarrow Y$. Namely, for any polymorphism $\mu: X \longmapsto Y$ there is a canonical map (defined a. s.) sending points $x \in X$ to conditional measures (see, e.g., 2], Sect. 10.4) $\mu_{x}(y)$ on $Y$ such that for any subsets $A \subset X, B \subset Y$ of finite measure we have

$$
\mu(A \times B)=\int_{A} \mu_{x}(B) d \xi(x) .
$$

Notice that $\mu_{x}(Y) \leqslant 1$ for almost all $x \in X$, also we have

$$
\int_{X} \mu_{x}(B) d \xi(x) \leqslant \mu(B) .
$$

Let $\mu: X \longmapsto Y, \nu: Y \succ Z$ be polymorphisms. We define their product $\varkappa=\nu \mu: X \longmapsto Z$ in the terms of conditional measures:

$$
\varkappa_{x}=\int_{Y} \nu_{y} d \mu_{x}(y) .
$$

Thus we get a category whose objects are Lebesgue measure spaces and morphisms are polymorphisms.

For a polymorphism $\mu: X \longmapsto Y$ we define the adjoint polymorphism $\mu^{\square}$ : $Y \rightarrow X$ that is the same measure considered as a measure on $Y \times X$.

B. Linear operators DETERMined by polymorphisms. For a polymorphism $\mu: X \longmapsto Y$ we consider the sesquilinear form

$$
S_{\mu}: L^{2}(X, \xi) \times L^{2}(Y, v) \rightarrow \mathbb{C}
$$

defined by

$$
S_{\mu}(\varphi, \psi)=\int_{X \times Y} \varphi(x) \overline{\psi(y)} d \mu(x, y) .
$$

Applying the Cauchy-Bunyakovsky inequality and the definition of polymorphisms we get

$$
\left|S_{\mu}(\varphi, \psi)\right| \leqslant\|\varphi\|_{L^{2}(X, \xi)} \cdot\|\psi\|_{L^{2}(Y, v)} .
$$

Therefore there exists a bounded operator

$$
\Pi(\mu): L^{2}(Y, v) \rightarrow L^{2}(X, \xi)
$$

such that for all $\varphi \in L^{2}(X, \xi), \psi \in L^{2}(Y, v)$ we have

$$
S_{\mu}(\varphi, \psi)=\langle\varphi, \Pi(\mu) \psi\rangle_{L^{2}(X, \xi)} .
$$

By (1.4), operators $\Pi(\mu)$ are contractions, i. e.,

$$
\|\Pi(\mu)\| \leqslant 1 .
$$


The explicit expression for this operators is

$$
\Pi(\mu) \psi(x)=\int_{Y} \psi(y) d \mu_{x}(y),
$$

where $\mu_{x}$ are the conditional measures defined above.

REMARK. The last expression shows that $\Pi(\mu)$ sends nonnegative functions to nonnegative functions. Conversely, say that an operator $T: L^{2}(Y, \xi) \rightarrow$ $L^{2}(X, \xi)$ is a sub-Markov operator if it satisfies this property and $\|T\| \leqslant 1$. It is easy to show that any sub-Markov operator has the form $T=\Pi(\mu)$ for some polymorphism $\mu$. The measure $\mu$ is determined by the condition

$$
\mu(A \times B):=\left\langle I_{A}, T I_{B}\right\rangle_{L^{2}(X, \xi)},
$$

where $A \subset X, B \subset Y$ are sets of finite measure.

REMARK. We also can describe the operator $\Pi(\mu)$ in the following way. Consider a bounded nonnegative function $\psi$ on $Y$ and the measure

$$
\psi(y) \mu(x, y)
$$

on $X \times Y$. Taking its projection to $X$ we get a measure, say $\Phi$, on $X$. For each measurable subset $A \subset X$ we have $\Phi(A)=S_{\mu}\left(I_{A}, \psi\right)$, where $I_{A}$ is the indicator function. Clearly for a set $C \subset X$ of zero measure we have $\Phi(C)=0$. Therefore, the measure $\Phi$ is absolutely continuous with respect to $\xi$, and we can define $\Pi(\mu) \psi$ as the Radon-Nikodym derivative $d \Phi / d \xi$, for formulas for Radon-Nikodym derivatives, see, e.g., 33, Sect. 10.

Formula (1.5) easily implies that

$$
\Pi(\nu \mu)=\Pi(\nu) \Pi(\mu) .
$$

So we get a functor from the category of polymorphisms to the category of Hilbert spaces and bounded operators.

C. Topology on sets $\operatorname{Pol}(X, Y)$. Next, let $\mu_{j}, \mu$ be polymorphisms $X \longmapsto$ $Y$. We say that $\mu_{j}$ converges to $\mu$ if for any subsets $A \subset X, B \subset Y$ of finite measure the sequence $\mu_{j}(A \times B)$ converges to $\mu(A \times B)$. It is easy to show that this convergence is equivalent to the weak operator convergence of the corresponding sub-Markov operators $\Pi\left(\mu_{j}\right) \rightarrow \Pi(\mu)$, i. e.,

$$
\left\langle\varphi, \Pi\left(\mu_{j}\right) \psi\right\rangle_{L^{2}(X, \xi)} \rightarrow\langle\varphi, \Pi(\mu) \psi\rangle_{L^{2}(X, \xi)} \quad \text { for all } \varphi \in L^{2}(X, \xi), \psi \in L^{2}(Y, v) .
$$

Let $H, K$ be separable Hilbert spaces. Denote by $\mathcal{C}(H, K)$ the set of all contractions $H \rightarrow K$, equip it with the weak operator topology. This set is compact metrizable and the multiplications

$$
\mathcal{C}(H, K) \times \mathcal{C}(K, L) \rightarrow \mathcal{C}(H, L)
$$

are separately continuous. This easily implies that sets $\operatorname{Pol}(X, Y)$ are compact metrizable and the product is separately continuous. 
D. Measure preserving transformations and polymorphisms. Let $(X, \xi)$ be a space with a $\sigma$-finite non-atomic measure (i.e., let $X$ be equivalent to $\mathbb{R})$. Denote by $\operatorname{Ams}(X)$ the group of measure preserving transformations of $X$. Let $g \in \operatorname{Ams}(X)$. Consider the map $X \rightarrow X \times X$ given by $x \mapsto(x, g(x))$, consider the image $\varkappa_{g}$ of the measure $\xi$ under this map. It is clear that $\varkappa_{g}$ is a polymorphism $X \longmapsto X$ and products of measure preserving transformations correspond to products polymorphisms. Also it is easy to show that the group $\operatorname{Ams}(X)$ is dense in the semigroup $\operatorname{Pol}(X, X)$.

So the notion of a polymorphisms extends the notion of measure preserving transformation (apparently, this idea arises to E. Hopf [8]).

E. References on POLYMorphisms. Polymorphisms and various variants of 'Markov operators' are the standard objects of ergodic theory, see, e.g., E. Hopf [8], J. Neveu [28], A. V. Vershik [37, U. Krengel [12]. There are several natural groups of transformation of measure spaces (measure preserving transformations of spaces with finite measures or with $\sigma$-finite measures, groups of regular transformations, etc.). For this reasons there are several kinds of 'polymorphisims', see [18, 19, Sect. VIII.4 and Chapter X. The version discussed above corresponds to the group of measure preserving transformations of a space with infinite continuous measure, apparently it appeared in [18.

K. Schmidt and A. V. Vershik 32 considered polyhomomorphisms ('algebraic polymorphisms') of compact groups $K$ under stronger conditions. In our terminology they consider polyhomomorphisms $R^{\circ}: K^{\circ} \rightarrow K^{\circ}$ such that $\operatorname{dom} R=K, \operatorname{im} R=K$ (also $\alpha\left(R^{\circ}\right)=\beta\left(R^{\circ}\right)=1$, but the last condition in this case is not essential). In particular, this includes the case of tori discussed above in Subsect. 1.3 .

1.6. Polymorphisms and polyhomomorphisms. So any polyhomomorphism is a polymorphism.

Theorem 1.5 The product of polyhomomorphism defined above corresponds to the product of polymorphisms.

The proof occupies Subsect. 2.2 2.3

This immediately implies the associativity of the product of polyhomomorphisms (Lemma 1.3) and the separate continuity (Proposition 1.4.b).

1.7. Linear operators determined by polyhomorphisms. Let $G$ be a locally compact group, $\Phi$ an open subgroup, and $\Delta$ a compact normal subgroup in $\Phi$. Denote by $L^{2}(\Phi)^{\Delta} \subset L^{2}(G)$ the subspace consisting of functions that are supported by $\Phi$ and invariant with respect to $\Delta$. Denote by $P_{\Phi \mid \Delta}^{G}$ the operator of orthogonal projection to the subspace $L^{2}(\Phi)^{\Delta} \subset L^{2}(G)$.

Proposition 1.6 Let $R^{\circ} \in \operatorname{Polh}\left(G^{\circ}, H^{\circ}\right)$. Then the operator

$$
\widetilde{\Pi}\left(R^{\circ}\right):=\left(\alpha\left(R^{\circ}\right) \beta\left(R^{\circ}\right)\right)^{-1 / 2} \Pi\left(R^{\circ}\right): L^{2}(H) \rightarrow L^{2}(G)
$$


is a partial isometry $5^{5}$. The initial subspace of $\widetilde{\Pi}\left(R^{\circ}\right)$ is $L^{2}(\operatorname{im} R)^{\text {indef } R}$, the final subspace is $L^{2}(\operatorname{dom} R)^{\operatorname{ker} R}$.

Explicit description of operators $\Pi\left(R^{\circ}\right)$ is contained in Subsect. 2.4.

So operators $\Pi\left(R^{\circ}\right)$ are 'partial homotheties'. By Theorem 1.5, a product $\Pi\left(T^{\circ}\right) \Pi\left(R^{\circ}\right)$ of two 'partial homotheties' is a 'partial homothety' again, but the new scaling coefficient is not a product of scaling coefficients. Formulate a geometric statement related to this phenomenon.

Let $L, M$ be two closed subspaces in a Hilbert space $H$. Consider the operators $P_{L}$ and $P_{M}$ of orthogonal projections $H \rightarrow L, H \rightarrow M$. Consider the self-adjoint operators

$$
\left.P_{L} P_{M}\right|_{L}=\left.P_{L} P_{M} P_{L}\right|_{L}: L \rightarrow L,\left.\quad P_{M} P_{L}\right|_{M}=\left.P_{M} P_{L} P_{M}\right|_{M}: M \rightarrow M .
$$

It is easy to see that their spectral types coincide upto multiplicities of zeros, and these spectral types are invariants of a pair of subspaces under unitary transformations (this is an analog of angles in elementary geometry, see, e.g., [21, Sect. 2.5).

Proposition 1.7 Let $G$ be a unimodular locally compact group, $\Phi, \Psi$ open subgroups, $\Delta$ be a compact normal subgroup in $\Phi, \Gamma$ a compact normal subgroup in $\Psi$. Let the pairs $(\Phi, \Delta)$ and $(\Psi, \Gamma)$ be different. Then the spectrum of the operator

$$
\left.P_{\Phi \mid \Delta}^{G} P_{\Psi \mid \Gamma}^{G}\right|_{L^{2}(\Phi)^{\Delta}}: L^{2}(\Phi)^{\Delta} \rightarrow L^{2}(\Phi)^{\Delta}
$$

consists of two point 6 , namely, 0 and

$$
\sigma:=([\Delta:(\Delta \cap \Psi)] \cdot[\Gamma:(\Gamma \cap \Phi)])^{-1} .
$$

Therefore the operator

$$
\left.\sigma^{-1 / 2} P_{\Psi \mid \Gamma}^{G}\right|_{L^{2}(\Phi)^{\Delta}}: L^{2}(\Phi)^{\Delta} \rightarrow L^{2}(\Psi)^{\Gamma}
$$

is a partial isometry.

The proof is contained in 2.5

1.8. Rational polyhomomorphisms. Let $K_{1}, K_{2} \subset G$ be open compact subgroups in a locally compact group $G$. Then $K_{1} \cap K_{2}$ also is an open compact subgroup. The homogeneous space $K_{1} /\left(K_{1} \cap K_{2}\right)$ is discrete and compact, therefore it is finite. Therefore the ratio of measures of $K_{1}$ and $K_{2}$ is rational.

\footnotetext{
${ }^{5}$ Consider Hilbert spaces $V, W$. A bounded linear operator $A: V \rightarrow W$ is called a partial isometry, if there exists a subspace $L \subset V$ (the initial subspace) and $M \subset W$ (the final subspace) such that the restriction of $A$ to $V$ is a unitary operator $L \rightarrow M$ and restriction of $A$ to the orthogonal complement $L^{\perp} \subset V$ is zero.

${ }^{6}$ This property is similar to isoclinicity. A pair of subspaces $L, M$ of a finite dimensional Euclidean space is called isoclinic, if the operator $P_{L} P_{M}: L \rightarrow M$ is scalar (or, equivalently, all Jordan angles between $L, M$ are equal), see, e.g. [39].
} 
Now we define a subcategory Polh $_{\mathbb{Q}}$ of the category of polymorphisms. Let us consider only unimodular locally compact groups that have open compact subgroups and equip them with Haar measures such that measures of open compact subgroups are rational. A rational polyhomomorphism $R^{\circ}: G^{\circ} \longmapsto H^{\circ}$ is a polyhomomorphism such that $\alpha\left(R^{\circ}\right), \beta\left(R^{\circ}\right)$ are rational. Since indices in formulas (1.1) - 1.2) are integer, products of rational polyhomomorphisms are rational.

1.9. Polyendomorphisms. Now consider a unimodular locally compact group $G$ containing an open compact subgroup $K_{0}$. Normalize the Haar measure on $G$ assuming that the measure of $K_{0}$ is 1 . For any pair $K_{1} \supset K_{2}$ of open compact subgroups consider the index $\left[K_{1}: K_{2}\right]$. Consider the multiplicative semigroup $\Lambda=\Lambda(G) \subset \mathbb{N}$ consisting of all products of such indices. Denote by $\operatorname{Polh}_{\Lambda}\left(G^{\circ}, G^{\circ}\right)$ the semigroup of all polyhomomorphisms $G^{\circ} \longmapsto G^{\circ}$ consisting of 0 and all $R^{\circ}$ such that $\alpha\left(R^{\circ}\right)^{-1}, \beta\left(R^{\circ}\right)^{-1} \in \Lambda$.

Theorem 1.8 The set $\operatorname{Polh}_{\Lambda}\left(G^{\circ}, G^{\circ}\right)$ is a compact subsemigroup in $\operatorname{Polh}\left(G^{\circ}, G^{\circ}\right)$.

The proof is contained in Subsect. 2.6.

1.10. Example: the group of infinite matrices over a finite field. Let $p$ be prime, $\mathbb{F}_{p}$ be the field with $p$ elements. Consider an infinite dimensional locally compact linear space over $\mathbb{F}_{p}$ satisfying the second county axiom. There are only 3 such spaces (this is semi-obvious, we present a formal proof below in Subsect. 2.7). The first space $\mathbb{V}_{p}^{+}$is a direct sum of a finch number of copies of the field $\mathbb{F}_{p}$ equipped with a discrete topology. The second space $\mathbb{V}_{p}^{-}$is the direct product of a countable number of copies of the field $\mathbb{F}_{p}$, it is equipped with the Tikhonov topology. These spaces are Pontryagin dual one to another (on the duality, see. e.g., [7, Chapter 6). The third space $\mathbb{V}_{p}:=\mathbb{V}_{p}^{-} \oplus \mathbb{V}_{p}^{+}$is the topic of our interest.

Consider the linear space $\mathbb{V}_{p}$ over $\mathbb{F}_{p}$ consisting of two-side sequences

$$
v=\left(\ldots, v_{-2}, v_{-1}, v_{0}, v_{1}, v_{2}, \ldots\right), \quad v_{k} \in \mathbb{F}_{p},
$$

such that $v_{j}=0$ for sufficiently large $j$. For each $m \in \mathbb{Z}$ consider the subspace $W^{m} \subset \mathbb{V}_{p}$ consisting of vectors $v$ such $v_{l}=0$ for all $l>-m$,

$$
\cdots \supset W^{-1} \supset W^{0} \supset W^{1} \supset W^{2} \supset \ldots
$$

The topology in $\mathbb{V}_{p}$ is defined by the condition: the subgroups $W^{m}$ are open and form a base of neighborhoods of 0 . A sequence $v^{(l)} \in \mathbb{V}_{p}$ converges to $v$ if it is contained in some subgroup $W^{m}$ and converges to $v$ coordinate-wise.

The subgroups $W^{m}$ are compact and are isomorphic to the countable direct product of cyclic groups $\mathbb{Z}_{p}$, quotients $\mathbb{V}_{p} / W^{m}$ are discrete and are isomorphic to the countable direct sum of of cyclic groups $\mathbb{Z}_{p}$. We normalize the Haar measure $\varphi(v)$ on $\mathbb{V}_{p}$ assuming that the measure of $W^{0}$ is 1 .

Consider groups $\mathrm{GL}\left(\mathbb{V}_{p}^{+}\right), \mathrm{GL}\left(\mathbb{V}_{p}^{-}\right), \mathrm{GL}\left(\mathbb{V}_{p}\right)$ of all continuous linear operators in these spaces. The representation theory of $\mathrm{GL}\left(\mathbb{V}_{p}^{+}\right)$is relatively simple (see 
T. Tsankov [36, it is not difficult to reduce this classification to a result of G. I. Olshanski in [30]), the group $\mathrm{GL}\left(\mathbb{V}_{p}^{-}\right)$is isomorphic to $\mathrm{GL}\left(\mathbb{V}_{p}^{+}\right)$. The group $\mathrm{GL}\left(\mathbb{V}_{p}\right)$ was introduced in [23], the representation theory of this group is non-trivial, see [23, 26], 27, it has many analogues with the representation theory of infinite-dimensional real classical groups in the sense of G. I. Olshanski 29.

Denote by $\operatorname{GL}\left(\mathbb{V}_{p}\right)$ the group of all continuous linear operators in $\mathbb{V}_{p}$, we can also say that it is the group $\operatorname{Aut}\left(\mathbb{V}_{p}\right)$ of continuous automorphisms of the Abelian group $\mathbb{V}_{p}$.

Denote by $J$ the operator of left shift of sequences (1.7). Clearly, this transformation sends the Haar measure $\varphi(v)$ to the measure $p \cdot \varphi(v)$. Denote by $\mathrm{GL}^{0}\left(\mathbb{V}_{p}\right)$ the subgroup of $\mathrm{GL}\left(\mathbb{V}_{p}\right)$ consisting of transformations preserving the Haar measure on $\mathbb{V}_{p}$. Clearly, the group $\mathrm{GL}\left(\mathbb{V}_{p}\right)$ is a semidirect product of the cyclic subgroup generated by $J$ and the normal subgroup $\mathrm{GL}^{0}\left(\mathbb{V}_{p}\right)$.

We have a measure preserving action of $\mathrm{GL}^{0}\left(\mathbb{V}_{p}\right)$ by automorphisms of the locally compact group $\mathbb{V}_{p}$, i.e., we are in the situation discussed in Subsect. 1.9] The semigroup $\Lambda\left(\mathbb{V}_{p}\right)$ consists of powers $p^{j}$, where $j \geqslant 0$. Closed subgroups in $\mathbb{V}_{p} \times \mathbb{V}_{p}$ are linear subspaces in $\mathbb{V}_{p} \oplus \mathbb{V}_{p}$.

Theorem 1.9 The closure of $\mathrm{GL}^{0}\left(\mathbb{V}_{p}\right)$ in $\operatorname{Polh}\left(\mathbb{V}_{p}, \mathbb{V}_{p}\right)$ coincides with the semigroup $\operatorname{Polh}_{\Lambda}\left(\mathbb{V}_{p}, \mathbb{V}_{p}\right)$.

The proof is contained in Subsect. 2.8,

Theorem 1.10 Any unitary representation of the group $\mathrm{GL}^{0}\left(\mathbb{V}_{p}\right)$ admits a continuous extension to a representation of the semigroup $\operatorname{Polh}_{\Lambda}\left(\mathbb{V}_{p}, \mathbb{V}_{p}\right)$ compatible with the involution.

The proof is contained in Subsect. 2.9,

1.11. Problem of closure. Consider a unitary representation $\rho$ of a topological group $G$ in a Hilbert space $H$. Consider the set $\rho(G)$ of unitary operators and close it in the space of all bounded operators with respect to the weak operator topology. It can be readily checked that this closure $\overline{\rho(G)}$ is a compact semigroup. G. I. Olshanski, see, e.g. 30, showed that such semigroups can be interesting algebraic objects and an effective tool for investigation of unitary representations of infinite-dimensional groups $G$, see more in [19].

Now let a group $G$ acts by transformations of a measure space $X$. Then it acts in $L^{2}(X)$ and we have the same question about weak closure. On the other hand such questions can be reformulated in the terms of closures of groups in semigroups of polymorphisms, apparently, the first problem of this type (closure of an infinite-dimensional orthogonal group acting on a space of Gaussian measures) was solved by Nelson [17] (see, also [24, Sect. 12), for several actions of infinite-dimensional groups closures were described in [20, [22, 25]. Theorem 1.9 gives an additional example of this kind.

The problem of weak closure is not interesting for semisimple real or $p$-adic groups (usually, we get the one-point compactification, see [9]). 
On the other hand there are lot of interesting results about closures of ergodic measure preserving actions of Abelian groups as $\mathbb{Z}, \mathbb{R}, \mathbb{Z}^{n}$. For a generic (in the sense of Baire category) transformations such closures are huge and are related to the centralizer of a transformation in the semigroup of Markov operators, see [11, 6], 35]. For non-mixing actions the problem of weak closure usually is a difficult problems, see [5], some relatively simple cases for spaces of infinite measures were examined in [13, 31.

ExAmPLE. a) Equip the countable space $\mathbb{V}_{p}^{+}$(see the previous subsection) with the counting measure. It can be readily checked that the closure of $\mathrm{GL}\left(\mathbb{V}_{p}^{+}\right)$ in $\operatorname{Polh}\left(\mathbb{V}_{p}^{+}, \mathbb{V}_{p}^{+}\right)$consists of partial linear bijections $\mathbb{V}_{p}^{+} \rightarrow \mathbb{V}_{p}^{+}$equipped with counting measures. By [36], the semigroup of partial linear bijections acts in all unitary representations of $\mathrm{GL}\left(\mathbb{V}_{p}^{+}\right)$.

b) Equip the space $\mathbb{V}_{p}^{-}$with the probabilistic Haar measure. It is easy to show that the closure $\mathrm{GL}\left(\mathbb{V}_{p}^{-}\right)$in $\operatorname{Polh}\left(\mathbb{V}_{p}^{-}, \mathbb{V}_{p}^{-}\right)$consists of $R^{\circ}$ such that $\operatorname{dom} R=\mathbb{V}_{p}^{-}$, $\operatorname{im} R=\mathbb{V}_{p}^{-}$and the Haar measure on $R$ is probabilistic.

\section{Proofs}

2.1. Immediate corollories of the definition of polyhomomorphism. Proof of Proposition 1.2. Here we prove statements a)-d) of Proposition 1.2. Recall that $R$ is a subgroup in $G \times H$ equipped with a left invariant Haar measure.

Statement A. Let we have a locally compact group $N$, its closed normal subgroup $K$, and the quotient group $M$. Denote left invariant Haar measures on these groups by $\nu(n), \varkappa(k), \mu(m)$ respectively. For $n \in N$ denote by $\dot{n}$ its image in $M$. According 4, Proposition VII.2.10, we have the following integration formula

$$
\int_{N} f(n) d \nu(n)=\int_{M} \int_{K} f(\dot{n} k) d \varkappa(k) d \mu(m) .
$$

Suppose that $K$ is not compact. Consider the image of the measure $\nu(n)$ under the homomorphism $N \rightarrow M$. We wish to show that compact subsets $U$ in $M$ with nonempty interiors have infinite measures. Indeed, let $\widetilde{U} \subset N$ be the preimage of $U$. Applying the integration formula to the indicator function $I_{\widetilde{U}}$ we get $\infty$.

We apply this remark to the group $N=R$, its subgroup $K=$ indef $R$, and the quotient $M=\operatorname{dom} R$ and observe that the projection of the measure $\rho(r)$ to $G$ can be dominated by the Haar measure $\rho(g)$ only if indef $R$ is compact. To verify the compactness of $\operatorname{ker} R$, we take the group $N=R$, the subgroup $K=\operatorname{ker} R$ and the quotient group $M=\operatorname{im} R$.

Statement B. The image and the domain are open subgroupd7.

\footnotetext{
${ }^{7}$ The author thanks the reviewer, who proposed a proof more natural than the proof in the first version of the paper. Initially, the statement was derived from the following result of J. Mackey [16. Theorem 7.2]: Let $G$ be a locally compact group, $L$ be a subgroup being a Borel subset in $G$. Then the homogeneous space $G / L$ has a standard Borel structure if and only if
} 
The group $R$ is a countable union of compact sets, therefore its image $\operatorname{dom} R$ also is a countable union of compact sets, and therefore is a Borel set.

Let $A$ be a set of nonzero measure in a locally compact group. Then by [38], $\S 11$, the set $A A^{-1}$ contains a neighborhood of the unit. Hence a subgroup of nonzero measure contains a neighborhood of unit and therefore is open.

Statement C. The group $R$ is unimodular. We apply formula (2.1) to $N=R, K=\operatorname{indef} R, M=\operatorname{dom} R$. Since $\operatorname{dom} R$ is an open subgroup in a unimodular group $G$, it is unimodular. The subgroup indef $R$ is compact, therefore it is unimodular, moreover, the measure is preserved under all automorphisms of indef $R$. Formula (2.1) shows that $\int f\left(h g h^{-1}\right)=\int f(g)$ for all $h$, this implies that $R$ is unimodular.

Since $R$ / indef $R \simeq \operatorname{dom} R$, we get that $R$ is unimodular.

Statement D. Let $g \in \operatorname{dom} R$. Then there is $h \in H$ such that $(g, h) \in R$. The Haar measure in $R$ is invariant with respect to the left shift by $(g, h)$. Therefore its projection $\nu$ to $G$ is invariant with respect to shift by $g$. Therefore $\nu$ is a Haar measure on $\operatorname{dom} R$.

2.2. Support of a product of polymorphisms. Here we derive Lemma 2.2. which is used below in discussion of products of polyhomomorphisms.

Let $X, Y$ be compact separable metric spaces. We say that a relation $R$ : $X \rightrightarrows Y$ is bi-proper, if the projections $R \rightarrow X$ are $R \rightarrow Y$ proper maps.

An equivalent definition: a relation $R: X \rightrightarrows Y$ is bi-proper if

1) $R \subset X \times Y$ is a closed subset;

2) for any compact subset $A \subset X$ the set $R A \subset Y$ is compact;

3) for any compact subset $B \subset Y$ the set $R^{\square} B \subset X$ is compact.

Lemma 2.1 Let $R: X \rightrightarrows Y, S: Y \rightrightarrows Z$ be bi-proper relations. Then $S R$ is bi-proper.

Proof. It is sufficient to verify that $S R \subset X \times Z$ is closed. Let a sequence $\left(x_{j}, z_{j}\right) \in X \times Z$ converges to $\left(x^{\circ}, z^{\circ}\right)$. Then there is a sequence $y_{j} \in Y$, such that $\left(x_{j}, y_{j}\right) \in R,\left(y_{j}, z_{j}\right) \in S$. The set $\Xi \subset X$ consisting of the sequence $x_{j}$ and its limit $x^{\circ}$ is compact. Therefore the subset $R \Xi \subset Y$ is compact. This set contains the sequence $\left(x_{j}, y_{j}\right)$, therefore we can choose a convergent subsequence $\left(x_{j_{k}}, y_{j_{k}}\right)$. Denote its limit by $\left(x^{\circ}, y^{\circ}\right)$. By the closeness of $R$ this limit is contained in $R$. Clearly, $y_{j_{k}}$ converges to $y^{\circ}$, therefore $\left(y^{\circ}, z^{\circ}\right) \in S$. Hence $\left(x^{\circ}, z^{\circ}\right) \in S R$.

Let $X, Y$ be locally compact complete metric spaces equipped with measures $\xi, v$ respectively. We say that a polymorphism $\mu: X \longmapsto Y$ is bi-proper supported by $R$, if

1. $R: X \rightrightarrows Y$ is a bi-proper relation;

2. $\mu$ is supported by $R$, i.e., $\mu((X \times Y) \backslash R)=0$.

$L$ is closed. In our case the subgroup $\operatorname{dom} R$ has non zero measure, therefore $G / \operatorname{dom} R$ is at most countable. It easy to show that the Borel structure on this set is standard. 
Lemma 2.2 Let $X, Y, Z$ be locally compact complete metric spaces, $\xi, v, \zeta$ be measures on these subspaces. Let a polymorphism $\mu \in \operatorname{Pol}(X, Y)$ be bi-proper supported by $R, \nu \in \operatorname{Pol}(Y, Z)$ be bi-proper supported by $S$. Then the product $\varkappa:=\nu \mu$ is bi-proper supported by the product of relations $S R$.

First, we prove the following lemma:

Lemma 2.3 Let $(X, \xi),(Y, v)$ be locally compact complete metric spaces with measures. Let $\mu \in \operatorname{Pol}(X, Y)$ be a bi-proper polymorphism supported by $R \subset$ $X \times Y$. For a point $y_{0} \in Y$ consider the set $R^{\square} y_{0}$, i.e. the set of all $x \in X$ such that $\left(x, y_{0}\right) \in R$. Then for any neighborhood $U$ of the set $R^{\square} y_{0}$ there is a neighborhood $V$ of the point $y_{0}$ such that for any function $\varphi$ supported by $V$ the function $\Pi(\mu) \varphi$ has a support in $U$.

Proof of Lemma 2.3. Let find $\Pi(\mu) \varphi$ applying Remark from Subsect. 1.5. B. For this purpose we must project the measure $\psi(y) \mu(x, y)$ to the space $X$. Clearly, for a function $\varphi$ whose support is contained in a small neighborhood of $y_{0}$, the support of the measure $\psi(y) \mu(x, y)$ is contained in a small neighborhood of the set $\left(X \times y_{0}\right) \cap R$. The support of the projection of the measure $\psi(y) \mu(x, y)$ is contained in a small neighborhood of the set $R^{\square} y$.

Proof of Lemma 2.2. Let $\left(x_{0}, z_{0}\right) \notin S R$. We must show that for real functions $\varphi \in C_{c}(X)$ supported by a sufficiently small neighborhood $A$ of the point $x_{0}$ and $\theta \in C_{c}(Z)$ supported by a sufficiently small neighborhood $B$ of the point $z$, we have $\int_{X \times Z} \varphi(x) \theta(z) d \varkappa(x, z)=0$. Evaluating this expression, we get

$$
\begin{array}{r}
\int_{X \times Z} \varphi(x) \theta(z) d \varkappa(x, z)=\langle\varphi, \Pi(\varkappa) \theta\rangle_{L^{2}(X, \xi)} \\
=\langle\varphi, \Pi(\mu) \Pi(\nu) \theta\rangle_{L^{2}(X, \xi)}= \\
=\left\langle\Pi\left(\mu^{\square}\right) \varphi, \Pi(\nu) \theta\right\rangle_{L^{2}(Y, v)} .
\end{array}
$$

By Lemma 2.3 a support of the function $\Pi(\nu) \theta$ is contained in a small neighborhood of the set $S^{\square} z_{0}$, a support of the function $\Pi\left(\mu^{\square}\right) \varphi$ is contained in a small neighborhood of the set $R x_{0}$. The condition $\left(x_{0}, z_{0}\right) \notin S R$ is equivalent to $S^{\square} z_{0} \cap R x_{0}=\varnothing$. Therefore (2.2) is zero for functions $\varphi, \theta$ with sufficiently small supports.

Other statements follow from Lemma 2.1.

2.3. The product of polyhomomorphisms. Thus, we have two polyhomomorphisms $R^{\circ}: G^{\circ} \longmapsto H^{\circ}, T: H^{\circ} \rightarrow K^{\circ}$. Notice that the indices

$$
\text { [ker } T:(\operatorname{ker} T \cap \operatorname{im} R)], \quad \text { indef } R:(\operatorname{indef} R \cap \operatorname{dom} T)
$$

in formulas (1.1) - (1.2) is finite. Indeed, the subgroup im $R$ is open (and therefore closed). Hence im $R \cap$ indef $T$ is open and closed in the compact group indef $T$. Therefore the quotient (indef $T) /(\operatorname{im} R \cap \operatorname{indef} T)$ is finite.

Next, we intend to evaluate the product of $R^{\circ}$ and $T^{\circ}$ as a product of polymorphisms and to verify that it coincides with the product $T^{\circ} R^{\circ}$ in the sense 
of polyhomomorphisms. By Lemma 2.2 the product is supported by the closed subgroup $R T$. We must show that the measure on $R T$ is a Haar measure.

Denote by $L_{G}(u)$ the transformation $v \mapsto u v$ on a group $G$. For the polyhomomorphism $R^{\circ}$ we have the following identity for polymorphisms:

$$
L_{H}(h) R^{\circ}=R^{\circ} L_{G}(g), \quad \text { for }(g, h) \in R .
$$

If also $(h, k) \in T$, then we have $T^{\circ} L_{H}(h)=L_{K}(k) T^{\circ}$ and therefore

$$
R^{\circ} T^{\circ} L_{G}(g)=L_{K}(k) R^{\circ} T^{\circ} .
$$

Therefore the polymorphism $R^{\circ} T^{\circ}$ is determined by a Haar measure on $R T$. It remains to find normalization constants $\alpha\left(T^{\circ} R^{\circ}\right), \beta\left(T^{\circ} R^{\circ}\right)$.

Lemma 2.4 Let $R^{\circ} \in \operatorname{Polh}\left(G^{\circ}, H^{\circ}\right)$, let $\Pi\left(R^{\circ}\right): L^{2}(H, \eta) \rightarrow L^{2}(G, \gamma)$ be the corresponding operator.

a) Let $B \subset \operatorname{im} R$ be a compact subset of nonzero measure invariant with respect to indef $R$. Then

$$
\Pi\left(R^{\circ}\right) I_{B}=\alpha\left(R^{\circ}\right) I_{R^{\square}} .
$$

b) Let $Z \subset$ indef $R$ be a subgroup of finite index $N$. Let $h_{1}, \ldots, h_{N}$ be representatives of double cosets - indef $R / Z$. Let $D \subset \operatorname{im} R$ be a compact set of non-zero measure invariant with respect to $Z$. Let the sets $h_{j} C$ are mutually disjoint. Then

$$
\Pi\left(R^{\circ}\right) I_{D}=\frac{\alpha\left(R^{\circ}\right)}{N} I_{R^{\square}} .
$$

Remarks. a) Since the subgroup indef $R$ is normal in im $R$, the left (indef $R$ )invariance of $B$ is equivalent to right (indef $R$ )-invariance.

b) For any compact $D$ we have

$$
\Pi\left(R^{\circ}\right) I_{D}=\Pi\left(R^{\circ}\right) I_{D \cap \mathrm{im} R} .
$$

Proof. a) We evaluate $\Pi\left(R^{\circ}\right) I_{B}$ using Remark in Subsect. 1.5 b. The measure (1.6) is supported by the set $M$ of all $(a, b) \in G \times H$ such that $(a, b) \in R$, $b \in B$ and coincides with the Haar measure $\rho(r)$ on this set. If $(a, b) \in M$ and $q \in$ indef $R^{\circ}$, then $(a, b q) \in M$. Therefore $M$ coincides with the preimage of $R^{\square} B$ under the projection $R \rightarrow G$. Projecting the Haar measure on $M$ to $G$ we get the measure $\alpha\left(R^{\circ}\right) \gamma$ restricted to $R^{\square} B$.

b) Obviously, for any set $D \subset H$ and $h \in$ indef $R$ the followin equality holds

$$
\Pi\left(R^{\circ}\right) I_{h D}=\Pi\left(R^{\circ}\right) I_{D} .
$$

Therefore, in our case all functions $\Pi\left(R^{\circ}\right) I_{h_{j} C}$ are equal. The sets $R^{\square}\left(h_{j} C\right)$ also coincide. Therefore

$$
\Pi\left(R^{\circ}\right) I_{C}=\frac{1}{N} \sum_{j} \Pi\left(R^{\circ}\right) I_{h_{j} C}=\frac{1}{N} \sum_{j} \Pi\left(R^{\circ}\right) I_{\cup h_{j} C} .
$$


Applying the statement a) to the last set, we come to

$$
\frac{\alpha\left(R^{\circ}\right)}{N} I_{R^{\square}\left(\cup h_{j} C\right)}=\frac{\alpha\left(R^{\circ}\right)}{N} I_{\cup R^{\square} h_{j} C}=\frac{\alpha\left(R^{\circ}\right)}{N} I_{R^{\square} C} .
$$

End of Proof of Theorem 1.5. Consider a compact subset $C \subset \operatorname{im}(T R)$ containing a neighbothood of the unit and invariant with respect to the subgroup

$$
\operatorname{indef}(T R)=T \text { indef } R \text {. }
$$

By virtue of Lemma 2.4 a,

$$
\Pi\left((T R)^{\circ}\right) I_{C}=\alpha\left((T R)^{\circ}\right) I_{R^{\square} T^{\square} C}
$$

By the same lemma and (2.3), we have

$$
\Pi\left(R^{\circ}\right) \Pi\left(T^{\circ}\right) I_{C}=\alpha\left(T^{\circ}\right) \Pi\left(R^{\circ}\right) I_{T^{\square} C}=\alpha\left(T^{\circ}\right) \Pi\left(R^{\circ}\right) I_{\mathrm{im} R \cap T^{\square}} .
$$

Generally, the set $\operatorname{im} R \cap T^{\square} C$ is not (indef $R$ )-invariant. However, by our choice of $C$, it is (indef $R \cap \operatorname{dom} T$ )- invariant. Applying Lemma 2.4 b to the last expression, we come to

$$
\frac{\alpha\left(T^{\circ}\right) \alpha\left(R^{\circ}\right)}{\text { [indef } R:(\operatorname{indef} R \cap \operatorname{dom} T)} I_{R^{\square} T^{\square} C} .
$$

The same considerations give us the constant $\beta\left(R^{\circ} T^{\circ}\right)$.

2.4. The description of operators $\Pi\left(R^{\circ}\right)$. Let $G$ be a locally compact group, $\Phi$ an open subgroup, and $\Delta$ a compact normal subgroup in $\Phi$. Normalize a Haar measure on the group $\Phi / \Delta$ as the image of $\left.\gamma(g)\right|_{\Phi}$ under the map $\Phi \rightarrow$ $\Phi / \Delta$. Consider the 'diagonal' map $\Phi \rightarrow \Phi \times(\Phi / \Delta)$ sending $g \in \Phi$ to $(g, g \Delta)$. We define the polyhomomorphism

$$
\mu_{G}^{\circ}[\Phi \mid \Delta] \in \operatorname{Polh}\left(G^{\circ},(\Phi / \Delta)^{\circ}\right)
$$

as the image of $\left.\gamma(g)\right|_{\Phi}$ under the 'diagonal' map (in particular $\alpha=\beta=1$ ).

On the other hand the map $\Phi \rightarrow \Phi / \Delta$ induces the operator

$$
\Pi\left(\mu_{G}^{\circ}[\Phi \mid \Delta]\right): L^{2}(\Phi / \Delta) \rightarrow L^{2}(\Phi) \subset L^{2}(G) .
$$

It is an isometric embedding $L^{2}(\Phi / \Delta) \rightarrow L^{2}(G)$ whose image is $L^{2}(\Phi)^{\Delta}$. The adjoint operator

$$
\Pi\left(\mu_{G}^{\circ}[\Phi \mid \Delta]^{\square}\right): L^{2}(G) \rightarrow L^{2}(\Phi / \Delta)
$$

can be described in the following way: we restrict a function $f \in L^{2}(G)$ to the open subgroup $\Phi$, take its average over the action of the compact group $\Delta$, and consider this average as a function on $\Phi / \Delta$.

Let $R^{\circ} \in \operatorname{Polh}\left[G^{\circ}, H^{\circ}\right]$. We decompose it as a product $R^{\circ}=T^{\circ} S^{\circ} Q^{\circ}$,

$$
G^{\circ} \stackrel{Q^{\circ}}{\longmapsto}(\operatorname{dom} R / \operatorname{ker} R)^{\circ} \stackrel{S^{\circ}}{\longmapsto}(\operatorname{im} R / \operatorname{indef} R)^{\circ} \stackrel{T^{\circ}}{\longmapsto} H^{\circ},
$$


where

$$
Q^{\circ}=\mu_{G}^{\circ}[\operatorname{dom} R \mid \operatorname{ker} R], \quad T^{\circ}=\mu_{H}^{\circ}[\operatorname{im} R \mid \text { indef } R]^{\square} .
$$

To define $S^{\circ}$ we consider the canonical map

$$
R \rightarrow(\operatorname{dom} R / \operatorname{ker} R) \times(\operatorname{im} R / \operatorname{indef} R)
$$

Its image is a graph of an isomorphism

$$
\Sigma:(\operatorname{dom} R / \operatorname{ker} R) \rightarrow(\operatorname{im} R / \operatorname{indef} R) .
$$

The Haar measure on this graph is the image of the measure $\rho(r)$.

The operators $\Pi\left(Q^{\circ}\right), \Pi\left(T^{\circ}\right)$ were described above in this subsection.

$$
\Pi\left(S^{\circ}\right) f(q)=\beta\left(R^{\circ}\right) \cdot f(\Sigma(q)), \quad \text { where } q \in \operatorname{im} R / \text { indef } R .
$$

Proof of Proposition 1.6. Thus, the operator $\Pi\left(R^{\circ}\right)$ decomposes into the product of three operators. The operators $\Pi\left(T^{\circ}\right)$ is the operator of projection

$$
\begin{aligned}
L^{2}(H) \simeq L^{2}(\operatorname{im} R)^{\text {indef } R} \oplus\left(L^{2}(\operatorname{im} R)^{\text {indef } R}\right)^{\perp} \rightarrow & \\
& \rightarrow L^{2}(\operatorname{im} R)^{\text {indef } R} \simeq L^{2}(\operatorname{im} R / \text { indef } R)
\end{aligned}
$$

Next, we apply the operator

$$
\Pi\left(S^{\circ}\right): L^{2}(\operatorname{im} R / \text { indef } R) \rightarrow L^{2}(\operatorname{dom} R / \operatorname{ker} R),
$$

which is unitary up to a scalar factor. The last operator is an isometric embedding

$$
\begin{aligned}
L^{2}(\operatorname{dom} R / \operatorname{ker} R) \simeq L^{2}(\operatorname{dom} R)^{\operatorname{ker} R} & \rightarrow \\
\rightarrow L^{2}(G) & \simeq L^{2}(\operatorname{dom} R)^{\operatorname{ker} R} \oplus\left(L^{2}(\operatorname{dom} R)^{\operatorname{ker} R}\right)^{\perp} .
\end{aligned}
$$

Clearly, the product is a partial isometry up to a scalar factor, the initial subspace is $L^{2}(\operatorname{im} R)^{\text {indef } R}$, the final subspace is $L^{2}(\operatorname{dom} R)^{\operatorname{ker} R}$.

2.5. The proof of Proposition 1.7. Recall that $\Phi, \Psi$ are open subgroups in $G, \Delta$ is a normal subgroup in $\Phi$, and $\Gamma$ is a normal subgroup in $\Psi$. Set

$$
V:=L^{2}(\Phi)^{\Delta} \quad \text { and } \quad W:=L^{2}(\Psi)^{\Gamma} \subset L^{2}(G)
$$

Denote by

$$
P:=P_{\Phi \mid \Delta}^{G}, \quad Q:=P_{\Psi \mid \Gamma}^{G}
$$

the projection operators to these subspaces. We wish to show that the selfadjoint operator

$$
\left.P Q\right|_{V}=\left.P Q P\right|_{V}: V \rightarrow V
$$

splits as a direct sum of a zero operator and a scalar operator. We can pass to the subspaces $V \ominus\left(V \cap W^{\perp}\right), W \ominus\left(W \cap V^{\perp}\right)$. Indeed, $Q$ is zero on $V \cap W^{\perp}$, 
and $W \cap V^{\perp}$ does not contained in the image of $Q$. Therefore, it is sufficient to show that $\left.P Q\right|_{V \ominus\left(V \cap W^{\perp}\right)}$ is a scalar operator.

Next, let a function $f \in V$ has a support in $\Phi \backslash \Psi$. Obviously, $Q f=0$. But $f \in V$ is $\Delta$-invariant, therefore a support of $f$ is actually contained in

$$
\Phi \backslash \Delta(\Phi \cap \Psi)=\Phi \backslash(\Phi \cap \Psi) \Delta .
$$

Therefore, without loss of a generality, we can assume that

$$
\Phi=\Delta \cdot(\Phi \cap \Psi), \quad \Psi=\Gamma \cdot(\Phi \cap \Psi) .
$$

Notice that (under this condition) $f \in V$ is determined by its value on $\Phi \cap \Psi$, and this restriction is $\Delta \cap \Psi$-invariant. Conversely, any $\Delta \cap \Psi$-invariant function from $L^{2}(\Phi \cap \Psi)$ admits an extension to the whole subgroup $\Phi$ by $\Delta$-invariance (and by 0 on $G \backslash \Phi)$. By the way, for two functions $f_{1}, f_{2} \in V$ the following identity holds

$$
\left\langle f_{1}, f_{2}\right\rangle_{L^{2}(G)}=\left\langle\left. f_{1}\right|_{\Phi},\left.f_{2}\right|_{\Phi}\right\rangle_{L^{2}(\Phi)}=[\Delta:(\Delta \cap \Psi)] \cdot\left\langle\left. f_{1}\right|_{\Phi \cap \Psi},\left.f_{2}\right|_{\Phi \cap \Psi}\right\rangle_{L^{2}(\Phi \cap \Psi)} .
$$

Similar statements take place for elements of the subspace $W$.

If $f \in V, h \in W$, then

$$
\langle f, h\rangle_{L^{2}(G)}=\left\langle\left. f\right|_{\Phi \cap \Psi},\left.h\right|_{\Phi \cap \Psi}\right\rangle_{L^{2}(\Phi \cap \Psi)}
$$

The operator $\left.Q\right|_{V}$ is described in the following way: we take a function $f \in V$ and average with respect to $\Gamma$ the function $\left.f\right|_{\Phi \cap \Psi}$. Point out that

$$
\left.Q f\right|_{\Phi \cap \Psi}=\frac{1}{[\Gamma:(\Gamma \cap \Phi)]}\left\{\text { average of }\left.f\right|_{\Phi \cap \Psi} \text { with respect to } \Gamma \cap \Phi\right\} .
$$

Notice, that the subgroups $\Delta \cap \Psi$ and $\Gamma \cap \Phi$ are normal in $\Phi \cap \Psi$, therefore the averaging with respect to $\Gamma \cap \Phi$ sends $\Delta \cap \Psi$-invariant functions to $\Delta \cap \Psi$-invariant functions.

Lemma 2.5 Let $f \in V \ominus\left(V \cap W^{\perp}\right)$. Then the function $\left.f\right|_{\Phi \cap \Psi}$ is invariant with respect to the subgroup $(\Delta \cap \Psi) \cdot(\Gamma \cap \Phi)$.

Proof. The space $V \cap W^{\perp}$ consists of functions $f$ such that the expression (2.4) is 0 . Therefore $V \ominus\left(V \cap W^{\perp}\right)$ consists of $\Gamma \cap \Phi$-invariant elements of $V$.

Proof of Proposition 1.7. For a function $f \in V \ominus\left(V \cap W^{\perp}\right)$ denote by $\tilde{f}$ its restriction to $\Phi \cap \Psi$. Formula (2.4) gives

$$
\left.Q f\right|_{\Phi \cap \Psi}=[\Gamma:(\Gamma \cap \Phi)]^{-1} \widetilde{f} .
$$


The similar formula for the operator $P$ leads to

$$
\left.P f\right|_{\Phi \cap \Psi}=[\Delta:(\Delta \cap \Psi)]^{-1}[\Gamma:(\Gamma \cap \Phi)]^{-1} \widetilde{f}
$$

and we get the desired statement.

2.6. Proof of Theorem 1.8 (semigroups $\operatorname{Polh}_{\Lambda}\left(G^{\circ}, G^{\circ}\right)$ are closed). Let a sequence of polyhomomorphisms $R_{j}^{\circ}=\left(R_{j}, \rho_{j}\right)$ containing in the semigroup $\operatorname{Polh}_{\Lambda}\left(G^{\circ}, G^{\circ}\right)$ converges to $R^{\circ}=(R, \rho) \in \operatorname{Polh}\left(G^{\circ}, G^{\circ}\right)$. We must show that $R^{\circ} \in \operatorname{Polh}_{\Lambda}\left(G^{\circ}, G^{\circ}\right)$.

By symbols $\rho_{j}, \rho$ we also denote the corresponding measures on $G \times G$. Let $R_{j}^{\circ} \in \operatorname{Polh}_{\Lambda}\left(G^{\circ}, G^{\circ}\right)$ converge to $R^{\circ} \in \operatorname{Polh}\left(G^{\circ}, G^{\circ}\right)$. Without loss of generality we can assume that the sequence $\alpha\left(R_{j}^{\circ}\right)$ converges (otherwise we pass to a subsequence). If it converges to 0 , then $R_{j}^{\circ}$ converges to the zero polyhomomorphism. Otherwise, $\alpha\left(R_{j}^{\circ}\right)$ is eventually constant, without loss of generality we can assume that it is constant. Also, we can assume that a sequence $\beta\left(R_{j}^{\circ}\right)$ is constant.

Consider a compact open subgroup $L \subset \operatorname{dom} R^{\circ}$ containing ker $R$. Denote $M:=R L$. Then $L \times M$ is a compact open subgroup in $G \times H$. We have

$$
\left.\rho\right|_{L \times M}=\left.\lim _{j \rightarrow \infty} \rho_{j}\right|_{L \times M}
$$

Next $\overleftarrow{\pi}\left(R_{j} \cap(L \times M)\right)$ is an open subgroup in $L$ of a certain index $p_{j}$. Without loss of generality we can assume that $p_{j}$ does not depend on $j$ or $p_{k} \rightarrow \infty$. In the second case we have

$$
\gamma\left(\overleftarrow{\pi}\left(\left.\rho_{j}\right|_{L \times M}\right) \leqslant \gamma\left(\overleftarrow{\pi}\left(\left.\rho_{j}\right|_{L \times H}\right)=\frac{\alpha}{p_{j}} \gamma(L)\right.\right.
$$

and $R_{j}^{\circ}$ converges to zero. So we consider the first case.

Groups $R_{j} \cap(L \times M)$ are open subgroups in $R_{j} \cap(L \times H)$ of indices $q_{j}$. Again, without loss of generality, we can assume that the sequence $q_{j}$ is constant. Now we have

$$
\gamma\left(\overleftarrow{\pi}\left(\left.\rho_{j}\right|_{L \times M}\right)\right)=\frac{\alpha}{p q} \gamma(L)
$$

Therefore, $\rho_{j}(L \times M)=\frac{\alpha}{p q} \gamma(L)$. Passing to the limit, we get $\rho(L \times M)=\frac{\alpha}{p q} \gamma(L)$ and $\alpha\left(R^{\circ}\right)=\frac{1}{p q} \alpha$.

2.7. Locally compact linear spaces over finite fields. Here we prove the statement about classification of locally compact linear spaces over the field $\mathbb{F}_{p}$, which was formulated at the beginning of Subsect. 1.10.

Thus, let $V$ be an infinite locally compact linear space over $\mathbb{F}_{p}$, equivalently, $V$ is a locally compact group such that $p \cdot v=0$ for all $v \in V$. Three cases are possible.

The first case. Let $V$ be discrete and therefore countable. Any countable linear space over $\mathbb{F}_{p}$ is isomorphic to $\mathbb{V}_{p}^{+}$.

The second case. Let $V$ be compact and infinite. Since $V$ is compact, the Pontryagin dual group $V^{\circ}$ is discrete. Therefore $V$ is dual to $\mathbb{V}_{p}^{+}$, i.e., $V \simeq \mathbb{V}_{p}^{-}$. 
The third case. Let $V$ by non-compact and non-discrete. For any character $\chi$ from $V$ to the multiplicative groups of complex numbers the identity $\chi(v)^{p}=1$ holds, i.e., values of $\chi$ have the form $e^{2 \pi i / p}$. According the Pontryagin duality, characters separate points of $V$. Hence $V$ is totally disconnected and therefore $V$ contains an open compact subgroup $W$ (see [7, Theorem 7.5]). If $W$ is finite, then $V$ is countable. If $V / W$ is finite, then $V$ is compact. So we can omit these cases. Thus, $W \simeq \mathbb{V}_{p}^{-}, V / W \simeq \mathbb{V}_{p}^{+}$. Next, we choose a basis $e_{j}$ in $V / W$ and choose representatives $\widetilde{e}_{j} \in V$. Then the linear span of vectors $\widetilde{e}_{j}$ is a discrete linear space in $V$ complementary to $W$.

2.8. The closure of $\mathrm{GL}^{0}\left(\mathbb{V}_{p}\right)$ in the semigroup of polyhomomorphisms. Here we prove Theorem 1.9 about the closure of the group $\operatorname{GL}^{0}\left(\mathbb{V}_{p}\right)$ in the semigroup of polyhomomorphisms of the space $\mathbb{V}_{p}$.

We must show that each element of $\operatorname{Polh}_{\Lambda}\left(\mathbb{V}_{p}, \mathbb{V}_{p}\right)$ is contained in the closure $\overline{\mathrm{GL}}$ of the group $\mathrm{GL}^{0}\left(\mathbb{V}_{p}\right)$. Point out that proofs in the present and next subsections are essentially based on results of [27].

For $m>0$ denote by $\theta_{m}: \mathbb{V}_{p} \longmapsto \mathbb{V}_{p}$ the linear relation consisting of $\left(v, v^{\prime}\right)$ such that $v_{j}=v_{j}^{\prime}=0$ for $j \geqslant m, v_{i}=v_{i}^{\prime}$ for $-m<i<m$, and $v_{j}, v_{j}^{\prime}$ are arbitrary if $v_{j} \leqslant-m$. Thus,

$$
\operatorname{ker} \theta_{m}=W^{m}, \quad \operatorname{indef} \theta_{m}=W^{m}, \quad \operatorname{dom} \theta_{m}=W^{-m}, \quad \operatorname{im} \theta_{m}=W^{-m},
$$

and

$$
\operatorname{dom} \theta_{m} / \operatorname{ker} \theta_{m} \simeq \mathbb{F}_{p}^{2 m-1} \simeq \operatorname{im} \theta_{m} / \operatorname{indef} \theta_{m},
$$

where $\mathbb{F}_{p}^{2 m-1}$ consists of vectors $\left(v_{-m+1}, v_{-m+2}, \ldots, v_{m-1}\right)$. The isomorphism $\operatorname{dom} \theta_{m} / \operatorname{ker} \theta_{m} \rightarrow \operatorname{im} \theta_{m} /$ indef $\theta_{m}$ is the identity map $\mathbb{F}_{p}^{2 m-1} \rightarrow \mathbb{F}_{p}^{2 m-1}$.

We define the polyhomomorphisms $\theta_{m}^{\circ} \in \operatorname{Polh}\left(\mathbb{V}_{p}, \mathbb{V}_{p}\right)$ assuming that $\alpha\left(\theta_{m}^{\circ}\right)=$ $\beta\left(\theta_{m}^{\circ}\right)=1$.

Lemma 2.6 a) $\theta_{m}^{\circ} \in \overline{\mathrm{GL}}$.

b) The sequence $\theta_{m}^{\circ}$ converges to the identical polyhomomorphism as $m$ tends to $\infty$.

Proof. Decompose the space $\mathbb{V}_{p}$ as a product of 3 measure spaces

$$
\mathbb{V}_{p}=V^{-} \times \mathbb{F}^{2 m-1} \times V^{+},
$$

where $V^{-}$consists of sequences $\left(\ldots, v_{m-1}, v_{m}\right)$, the $\mathbb{F}_{p}^{2 m-1}$ of vectors

$$
\left(v_{-m+1}, v_{-m+2}, \ldots, v_{m-1}\right),
$$

and $V^{+}$of vectors $\left(v_{m}, v_{m+1}, \ldots\right)$. The space $V^{+}$is countable and measures of all points are 1 . The space $\mathbb{F}_{p}^{2 m-1}$ is finite and measures of all points are $p^{-m}$. The space $V^{-}$is equipped by product of uniform probabilistic measures on $\mathbb{F}_{p}$. The measure of $W^{0}$ is 1 .

Consider the sequence

$$
S_{j}^{+}=\left(\begin{array}{ccc}
0 & 1_{j} & 0 \\
1_{j} & 0 & 0 \\
0 & 0 & 1_{\infty}
\end{array}\right)
$$


of linear transformations in $V^{+}$. Clearly, it converges in $\operatorname{Polh}\left(V^{+}, V^{+}\right)$to the delta measure supported by 0 .

Next, consider the sequence

$$
S_{j}^{-}:=\left(\begin{array}{ccc}
1_{\infty} & 0 & 0 \\
0 & 0 & 1_{j} \\
0 & 1_{j} & 0
\end{array}\right)
$$

of linear transformations of the space $V^{-}$. Clearly, in $\operatorname{Polh}\left(V^{-}, V^{-}\right)$it converges to the product measure on $V^{-} \times V^{-}$.

Let us regard $S_{j}^{+}$(resp. $S_{j}^{-}$) as polymorphisms of the whole $\mathbb{V}_{p}$. Then

$$
\lim _{j \rightarrow \infty} \lim _{i \rightarrow \infty} S_{i}^{+} S_{j}^{-}=\theta_{m}^{\circ}
$$

b) Consider the compact subgroup $W^{l}$ with $l>0$, a vector $v \in W^{-k}$, and the indicator function $I_{v+W^{l}}$. Then for $m>\max (l, k)$ we have $\Pi\left(\theta_{m}^{\circ}\right) I_{v+W^{l}}=$ $I_{v+W^{l}}$.

By the separate continuity of the product the statement a) implies the following corollary.

Lemma 2.7 For any $g \in \mathrm{GL}^{0}$ we have $\theta_{m}^{\circ} g \theta_{m}^{\circ} \in \overline{\mathrm{GL}}$.

Lemma 2.8 Let $R^{\circ} \in \operatorname{Polh}_{\Lambda}\left(\mathbb{V}_{p}, \mathbb{V}_{p}\right)$. Then there exists $g \in \mathrm{GL}^{0}$ such that

$$
\theta_{m}^{\circ} R^{\circ} \theta_{m}^{\circ}=\theta_{m}^{\circ} g \theta_{m}^{\circ}
$$

Moreover, we can choose a finitary $g$, i.e., $g$ such that $g-1$ has only finite number of nonzero matrix elements.

Proof. Notice that for $Q_{m}^{\circ}:=\theta_{m}^{\circ} R^{\circ} \theta_{m}^{\circ}$,

$\operatorname{dom} Q_{m} \subset W^{-m}, \quad \operatorname{im} Q_{m} \subset W^{-m}, \quad \operatorname{ker} Q_{m} \supset W^{m}, \quad$ indef $Q_{m} \supset W^{m}$.

Therefore $Q_{m}^{\circ}$ determines a polyhomomorphism

$$
W^{-m} / W^{m} \longmapsto W^{-m} / W^{m},
$$

i.e, $\mathbb{F}^{2 m-1} \longmapsto \mathbb{F}^{2 m-1}$, measures on both copies of $\mathbb{F}^{2 m-1}$ are uniform, a measure of a point is $p^{-m}$.

In particular, we can apply this reasoning to $\theta_{m}^{\circ} g \theta_{m}^{\circ}$, where $g$ is finitary matrix. This polyhomomorphism determines a polyhomomorphism $\chi^{\circ}(g)$ : $\mathbb{F}^{2 m-1} \longmapsto \mathbb{F}^{2 m-1}$. The underlying linear relation $\chi(g): \mathbb{F}^{2 m-1} \rightrightarrows \mathbb{F}^{2 m-1}$ consists of $(u, v)$ such that there exist $x \in W^{m}, y \in W^{m}$ satisfying

$$
\left(\begin{array}{l}
x \\
u \\
0
\end{array}\right)\left(\begin{array}{lll}
g_{11} & g_{12} & g_{13} \\
g_{21} & g_{22} & g_{23} \\
g_{31} & g_{32} & g_{33}
\end{array}\right)\left(\begin{array}{l}
y \\
v \\
0
\end{array}\right) .
$$


This means that $\chi(g)$ is the characteristic linear relation of $g$ in the sense [27, Subsect. 1.5. Next, we must find the normalization of the Haar measure $\theta_{m}^{\circ} g \theta_{m}^{\circ}$. Evaluating $\alpha\left(\theta_{m}^{\circ} \cdot g \theta_{m}^{\circ}\right)$ by formula (1.1) we get

$$
\alpha\left(\chi^{\circ}(g)\right)=p^{-\operatorname{rk} g_{13}} .
$$

In notation of 27, Subsect. 1.5, $\operatorname{rk} g_{13}$ is the invariant $\eta(g)$. So we get a polyhomomorphism $\chi^{\circ}(g): \mathbb{F}^{2 p-1} \longmapsto \mathbb{F}^{2 p-1}$ such that measure of each point of $\chi(g)$ is $p^{-m-\mathrm{rk} g_{13}-\operatorname{dim} \text { indef } \chi(g)}$ and

$$
\beta\left(\chi^{\circ}(g)\right)=p^{-\operatorname{rk} g_{13}-\operatorname{dim} \operatorname{indef} \chi(g)+\operatorname{dim} \operatorname{ker}(g)} .
$$

Now consider an arbitrary linear relation $Q: \mathbb{F}^{2 m-1} \rightrightarrows \mathbb{F}^{2 m-1}$ and a polyhomomorphism $Q^{\circ}$ with $\alpha\left(Q^{\circ}\right)=p^{-\mu}$. Then

$$
\beta\left(Q^{\circ}\right)=\alpha\left(Q^{\circ}\right) p^{\operatorname{dim} \operatorname{ker} Q-\operatorname{dim} \operatorname{indef} P}
$$

We have $\beta \leqslant 1$. By [27, Proposition 1.8, any such polyhomomorphism can arise as $\chi^{\circ}(g)$ for a finitary $g$.

Lemma 2.9 For any $R^{\circ} \in \operatorname{Polh}\left(\mathbb{V}_{p}, \mathbb{V}_{p}\right)$ the sequence $Q_{m}^{\circ}:=\theta_{m}^{\circ} R^{\circ} \theta_{m}^{\circ}$ converges to $R^{\circ}$. Also

$$
\lim _{n \rightarrow \infty, m \rightarrow \infty} \theta_{m}^{\circ} R^{\circ} \theta_{n}^{\circ}=R^{\circ} .
$$

Proof. Fix $W_{k}$ and two vectors $v, w \in W_{-l}$. Clearly the sequence

$$
Q_{m}^{\circ}\left(\left(v+W_{k}\right) \times\left(w+W_{k}\right)\right)
$$

became constant after $m=\max (k, l)$.

Theorem 1.9 follows from Lemmas 2.7 2.9 .

2.9. Semigroup extensions of unitary representations of $\mathrm{GL}^{0}\left(\mathbb{V}_{p}\right)$. Below we give the proof of Theorem 1.10, it is based on 19, Theorem VIII.1.10.

We define the category $\overline{\mathcal{K}}$, whose objects are spaces $\mathbb{F}_{p}^{2 m-1}$ with Haar measure normalized as above and $\mathbb{V}_{p}$. Morphisms are polyhomomorphisms. We define the subcategory $\mathcal{K}$, whose objects are the spaces $\mathbb{F}_{p}^{2 m-1}$ with the same morphisms.

For any $m<n<\infty$ we define the linear relation $\lambda_{m n}: \mathbb{F}^{2 m-1} \rightrightarrows \mathbb{F}^{2 n-1}$ as the subspace consisting of vectors

$$
\left(v_{-m+1}, \ldots, v_{m-1}\right) \oplus\left(v_{-n+1}, \ldots, v_{-m}, v_{-m+1}, \ldots, v_{m-1}, 0, \ldots, 0\right) .
$$

We also define linear relations $\lambda_{m \infty}: \mathbb{F}^{2 m-1} \rightrightarrows \mathbb{V}_{p}$ consisting of vectors

$$
\left(v_{-m+1}, \ldots, v_{m-1}\right) \oplus\left(\ldots, v_{-m-1}, v_{-m}, v_{-m+1}, \ldots, v_{m-1}, 0,0 \ldots\right) .
$$

Define corresponding polyhomomorphisms $\lambda_{m n}^{\circ}, \lambda_{m \infty}^{\circ}$ assuming that all $\alpha(\cdot)$, $\beta(\cdot)$ are 1. Define adjoint polyhomomorphisms $\mu_{m n}^{\circ}:=\left(\lambda_{m n}^{\circ}\right)^{\square}, \mu_{m \infty}^{\circ}=\left(\lambda_{m \infty}^{\circ}\right)^{\square}$. 
It can be readily checked that we get a structure of an ordered category in the sense of [19], Sect. III.4.

By [27 any unitary representation of $\mathrm{GL}^{0}\left(\mathbb{V}_{p}\right)$ generates a representation of the category $\mathcal{K}$. Our Lemma 2.9 allows to apply Approximation theorem VIII.1.10 from [19, this implies that any $*$-representation of the category $\mathcal{K}$ extends to a representation of $\overline{\mathcal{K}}$. In particular the representation of the group $\mathrm{GL}^{0}\left(\mathbb{V}_{p}\right)$ extends to a representation of $\operatorname{Polh}_{\Lambda}\left(\mathbb{V}_{p}, \mathbb{V}_{p}\right)$.

\section{References}

[1] Biringer, I. Metrizing the Chabauty topology. Geom. Dedicata 195 (2018), $19-22$.

[2] Bogachev V. I. Measure theory. Vol. II. Springer-Verlag, Berlin, 2007.

[3] Bourbaki N. General topology. Chapters 1-4. Reprint of the 1989 English translation. Elements of Mathematics (Berlin). Springer-Verlag, Berlin, 1998

[4] Bourbaki N. Integration. II. Chapters 7-9. Translated from the 1963 and 1969. Elements of Mathematics (Berlin). Springer-Verlag, Berlin, 2004.

[5] Janvresse, É.; Prikhod'ko, A. A.; de la Rue, T.; Ryzhikov, V. V. Weak limits of powers of Chacon's automorphism. Ergodic Theory Dynam. Systems 35 (2015), no. 1, 128-141.

[6] Janvresse É, de la Rue Th., Ryzhikov V. Around King's rank-one theorems: flows and $\mathbb{Z}_{n}$-actions. in Dynamical systems and group actions (eds. Bowen L., Grigorchuk R., Vorobets Y.), 143-161, Contemp. Math., 567, Amer. Math. Soc., Providence, RI, 2012.

[7] Hewitt E., Ross K. A. Abstract harmonic analysis. Vol. I: Structure of topological groups. Integration theory, group representations. Academic Press, New York, 1963

[8] Hopf, E. The general temporally discrete Markoff process. J. Ration. Mech. Anal., 3 (1954) 13-45.

[9] Howe R. E., Moore C. C. Asymptotic properties of unitary representations. J. Funct. Anal. 32, 72-96 (1979).

[10] Kechris A. S. Classical descriptive set theory. Springer-Verlag, New York, 1995.

[11] King J. The commutant is the weak closure of the powers, for rank-1 transformations. Ergodic Theory Dynam. Systems 6 (1986), no. 3, 363-384.

[12] Krengel U. Ergodic theorems. Walter de Gruyter \& Co., Berlin, 1985. 
[13] Kushnir A. Yu., Ryzhikov V. V., Weak Closures of Ergodic Actions, Math. Notes, 101:2 (2017), 277-283.

[14] MacLane, S. An algebra of additive relations. Proc. Nat. Acad. Sci. USA, 47 (1961) 1043-1051.

[15] Michael E. Topologies on spaces of subsets. Trans. Amer. Math. Soc. 71 (1951), 152-182.

[16] Mackey G. W., Borel structure in groups and their duals. Trans. Amer. Math. Soc. 85 (1957), 134-165.

[17] Nelson E., The free Markoff field, J. Funct. Anal. 12 (1973) 211-227.

[18] Neretin Yu. A., Categories of bistochastic measures, and representations of some infinite-dimensional groups, Russian Acad. Sci. Sb. Math., 75:1 (1993), 197-219.

[19] Neretin Yu.A., Categories of Symmetries and Infinite-Dimensional Groups, Oxford University Press, New York, 1996.

[20] Neretin Yu. A., Spreading maps (polymorphisms), symmetries of Poisson processes, and matching summation. J. Math. Sci. (N. Y.), 126:2 (2005), 1077-1094.

[21] Neretin, Yu. Lectures on Gaussian integral operators and classical groups. European Mathematical Society (EMS), Zürich, 2011

[22] Neretin, Yu. Symmetries of Gaussian measures and operator colligations. J. Funct. Anal. 263 (2012), no. 3, 782-802.

[23] Neretin Yu. A. The space $L^{2}$ on semi-infinite Grassmannian over finite field. Adv. Math. 250 (2014), 320-350.

[24] Neretin Yu. A., Topological groups and invariant measures. Preprint, arXiv:1510.03082,

[25] Neretin Yu. A., Wishart-Pickrell distributions and closures of group actions, J. Math. Sci. (N. Y.), 224:2 (2017), 328-334.

[26] Neretin Yu. A., On the Weil representation of infinite-dimensional symplectic group over a finite field. Preprint https://arxiv.org/abs/1703.07238.

[27] Neretin Yu. A., Groups $\mathrm{GL}(\infty)$ over finite fields and multiplications of double cosets. Preprint https://arxiv.org/abs/2002.09969

[28] Neveu, J. Mathematical foundations of the calculus of probability. San Francisco, Holden-Day, Inc., 1965 
[29] Olshanski G.I., Unitary representations of infinite dimensional pairs $(G, K)$ and the formalism of R. Howe, in: Representation of Lie Groups and Related Topics (eds. Vershik A. M. and Zhelobenko D. P.), Gordon \& Breach 7 (1990) 269-463.

[30] Olshanski G.I., On semigroups related to infinite-dimensional groups, in: A.A. Kirillov (Ed.), Topics in Representation Theory, Amer. Math. Soc., Providence, RI, 1991, pp. 67-101.

[31] Ryzhikov V. V. Weak Closure of Infinite Actions of Rank 1, Joinings, and Spectrum, Math. Notes, 106:6 (2019), 957-965.

[32] Schmidt K., Vershik A. Algebraic polymorphisms. Ergodic Theory Dynam. Systems 28 (2008), no. 2, 633-642.

[33] Shilov G. E., Gurevich B. L. Integral, measure and derivative: A unified approach. Prentice-Hall, Inc., Englewood Cliffs, N.J. 1966

[34] Schubert H. Categories. Berlin-Heidelberg-New York: Springer-Verlag, 1972.

[35] Solecki S. Closed subgroups generated by generic measure automorphisms. Ergodic Theory Dynam. Systems 34 (2014), no. 3, 1011-1017.

[36] Tsankov, T. Unitary representations of oligomorphic groups. Geom. Funct. Anal. 22 (2012), no. 2, 528-555.

[37] Vershik A. M., Many-valued measure-preserving mappings (polymorphisms) and Markovian operators, J. Soviet Math., 23:3 (1983), 2243-2266.

[38] Weil, A. L'intǵration dans les groupes topologiques et ses applications. (French) Hermann et Cie., Paris, 1940.

[39] Wolf J. A., Elliptic spaces in Grassmann manifolds. Ill. J. Math., 7 (1963) 447-462.

[40] Zhelobenko, D. P. Principal structures and methods of representation theory. Providence, RI, American Mathematical Society (AMS), 2006

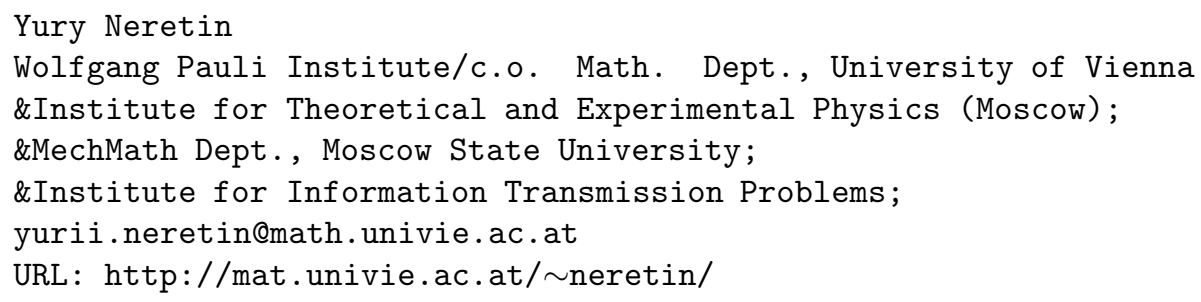

